\title{
The neuroleptics: a historical survey
}

Deniker P. The heuroleptics: a historical suŕvey. Acta Psychiatr Scand 1990; 82 (Suppl.358): 83-87.

Abstract: The history of the neuroleptic drugs has been marked by various milestones: some have been successes, others have been associated with complications and side effects, especially when the drugs have been misused. At the Paris meeting in 1955, it was confirmed that neuroleptics were indicated in chronic psychoses. In 1963, a new form of long-acting neuroleptic was introduced which appeared useful for non-compliant patients. However, following the introduction of the American concept of "CPZ- equivalent" which is opposed to the European distinction between sedative and "disinhibitory" neuroleptics, the problem of the development of tardive dyskinesia became serious. The antipsychotic actions of the neuroleptics in some psychoses eventually led to the so-called "dopaminergic theories" of schizophrenia, which are still under discussion. It should be underlined that the specific antimanic action of neuroleptics, which is included in the early definition, has been prematurely neglected. In fact, mania is the opposite of depression and, in general, the antidepressants are "anti-neuroleptic". The neurobiological studies of the neuroleptics resulted in the differentiation of the compounds according to their action on different parts of the dopamine system, especially on the mesolimbic and mesocortical structures. This was particularly useful in the efforts to avoid extrapyramidal side effects. The clarification of the role of the $D_{1}$ and $D_{2}$ dopamine receptors has been important in understanding the development of tardive dyskinesias. There are now some 50 different neuroleptic drugs, in about a dozen chemical families, which can be classified by an "automatic" statistical method.
P Deniker
Hospital Sainte-Anne, Parí, France

Key words: neuroleptics - psychoses schizophrenia - dopamine.

Pierre Deniker, M.D., French Academy of Medicine, Consultant, Hospital Sainte-Anne, 1 rue Cabanis, F 75014 Paris, France
The history of the neuroleptics has been marked by a number of distinct phases since its inception in 1952 and the recent introduction of several new compounds.

\section{The beginnings}

Chlorpromazine (CPZ) was the leading neuroleptic the name given to this class of drug in 1955. It was discovered in France and its psychiatric applications were investigated in 1952 in Europe and in 1954 in America. Reserpine, a completely different chemical entity, was introduced into psychiatry almost simultaneously in 1954 in France, Switzerland and America. The indications for both drugs in the psychoses were similar. Their effects on sleep and the autonomic nervous system had already been observed, but Steck was the first to describe the extrapyramidal side effects.

The first international conferences were held in Paris and Milan in October 1955, and there the indications for and the results of treatment with both drugs were reviewed, confirming that they were indicated for chronic psychoses. At the first meeting on psychotropic drugs, held in Milan in May 1957 (just preceding the birth of the CINP), Delay and Deniker presented a pharmacoclinical definition of neuroleptics based on the common similarities that differentiated the early com- pounds from all other psychotropic drugs. We underlined the fact that the psychomotor aspects of the symptomatology, generally reversible with drugs, were of the same nature as those observed in epidemic encephalitis by eminent neurologists like P. Marie, Marikasco, J. Lhermitte et al. and his colleagues.

Over the next few years, other compounds were discovered in other chemical groups: the butyrophenones produced haloperidol (1958) which possesses a specific anti-hallucinatory property, and the benzamides yielded sulpiride (1965), a derivative of metoclopramide, an anti-emetic drug. Since then, a dozen or more chemical families have become involved and more than $50 \mathrm{com}-$ pounds have been proposed as so-called antipsychotics. In each group the formulas that seem to indicate activity also produce, to a greater or lesser extent, psychomotor, autonomic, or extrapyramidal side effects. But an important milestone was the discovery of a common antidopaminergic activity of the effective drugs.

\section{Anti-dopaminergic activity}

Carlsson, in 1963, showed that, of all the different chemically related compounds, only those with an antipsychotic action blocked the postsynaptic dopamine neurones. The exception which proved the rule was 


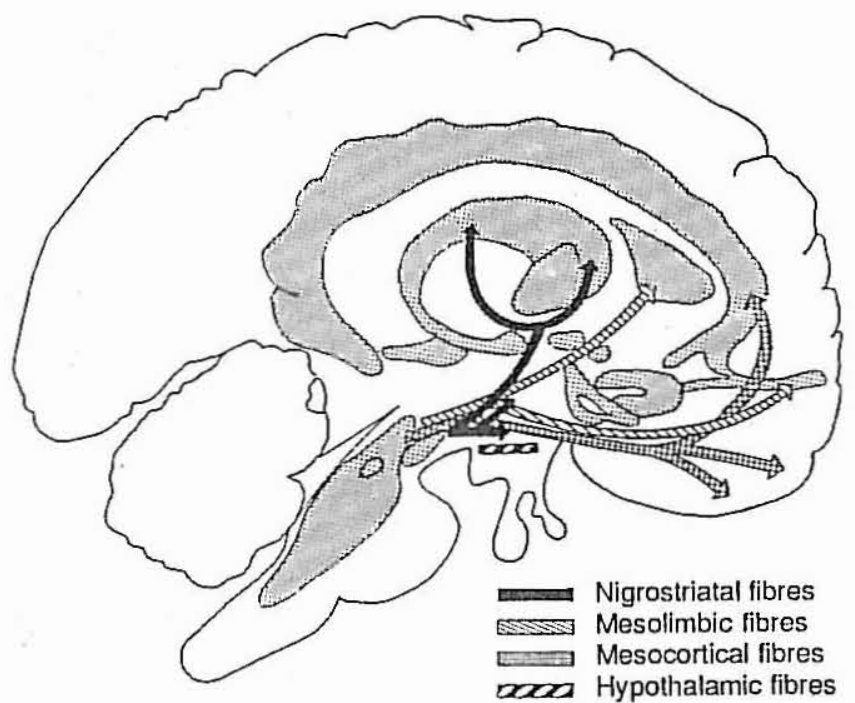

Fig.1. The dopaminergic system.

reserpine, but by depleting all amines it achieved the same result. Progress in our knowledge of the dopaminergic structures in the brain led to an ability to explain and understand the behavioural differences of the neuroleptics (Figs 1 and 2). In this particular field, the observations of the clinicians are in complete agreement with the experiences of the neurochemists and this should help to solve the problem of extrapyramidal side effects and their incidence in the chemotherapy of psychoses.

The impact of drugs on the different parts of the dopamine system has led to a search for drugs acting especially on the limbic and meso-cortical structures.

\section{Chemotherapy in practice}

Adoption of the new psychiatric drugs was slow at first. In France, the use of chlorpromazine in the 96 psychiatric hospitals increased from $0.428 \mathrm{~kg}$ to $2.33 \mathrm{~kg}$ between 1952 and 1957, and of reserpine from $33 \mathrm{~g}$ to

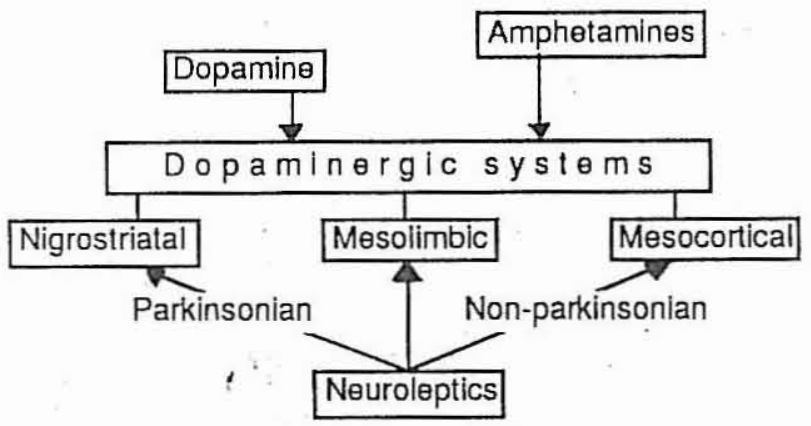

Fig.2. Dopaminergic and anti-dopaminergic actions of psychiatric agents.
$39 \mathrm{~kg}$. But in 1956 one hospital hardly used these drugs at all.

In 1958, at the first CINP meeting, Sir Aubrey Lewis of London declared: "Certainly if we had to choose between abandoning the use of all the new psychotropic drugs and abandoning the Industrial Resettlement Units and other social facilities available to us, there would be no hesitation about the choice: drugs would go". Fortunately, this choice did not have to be made.

At the same session, Brill, reporting on the New York State experience, said that in 1955 a large-scale therapeutic programme with chlorpromazine and reserpine had been undertaken on 25000 of the 93000 patients. He had noted a reduction of 3000 in the population instead of an expected increase of 8000.The number of patients treated rose to 30000 and in the same period the measures of restraint and confinement fell from 25 per thousand to 5 .

With the growing practice of chemotherapy, two important questions arose regarding the choice of drugs and their dosage. In France, the introduction of a new phenothiazine, prochlorperazine, which seemed to have some disinhibitory (that is to say, "stimulant") effect also raised the problem of therapeutic classification.

In Europe, different types of classification have been proposed since 1960; the last clinical one, in 1971, adopted a bipolar scheme and divided the compounds into four groups (Fig. 3).

However, in the United States, the concept of CPZequivalent made its appearance; supported in particular by Ban and Davis, this was generally adopted on the other side of the Atlantic.

As early as 1956, the question of why dosages were

Sedative action

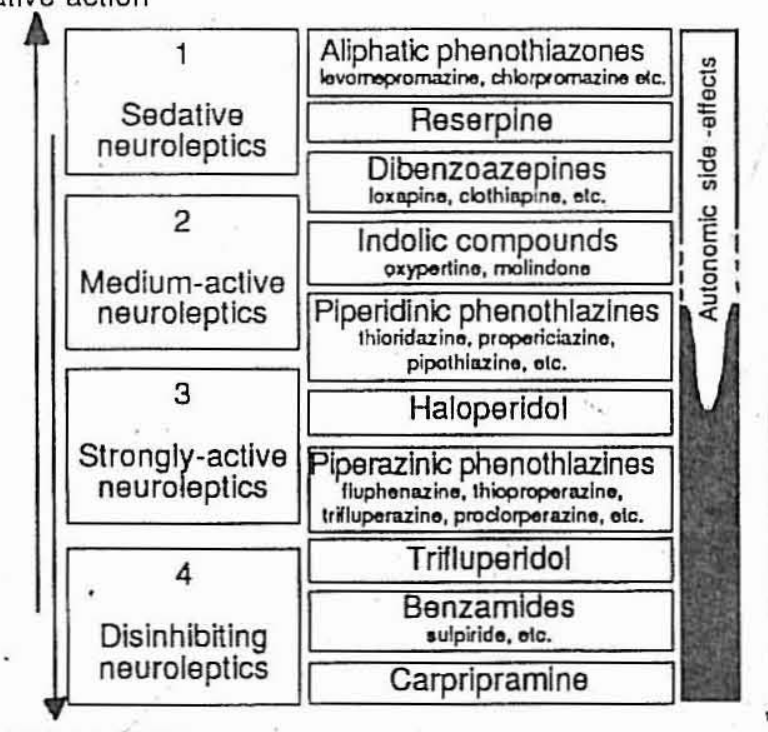

Disinhibiting action

Fig.3. The 1971, bipolar clinical classification of the neuroleptics which divided the compounds into four groups. 
higher in America than in Europe was posed. Denber was appointed by New York State and by SKF Company to investigate the reason. The answer was simple: American psychiatrists were more hurried than their European colleagues. Hoch and his colleagues showed that patients with psychoses who were treated with doses of up to 400 or $500 \mathrm{mg} /$ day of chlorpromazine did not respond any better than those treated with lower doses. This inflation of dosage plays a part, as we shall see, in the phenomenon of tardive dyskinesia.

\section{Adverse effects of neuroleptics}

From the beginning, neuroleptics had to face the consequences of adverse reactions. The first question put to us in the USA concerned the problem of jaundice with chlorpromazine. Later it was shown that the drug can induce microcanalicular obstruction of the biliary passages. Allergic reactions in nurses were avoided by adequate preparation of the tablets. A further impediment appeared with the so-called purple people. Some skin discolouration and ocular deposits occurred in patients who were photosensitive. This was the first adverse effect of high dosages to appear.

Last but not least, were the tardive dyskinesias which were responsible for many problems in the United States and led the Food and Drug Administration (FDA) to include all neuroleptics (except levomepromazine which is not registered as an antipsychotic but as an antihypertensive drug) on a waming list in 1985.

To try to explain these unexpected complications we have drawn attention to several factors that may possibly be involved.

First the posology: in the survey of Jeste and Wyatt (1981) concerning 12000 patients studied over 20 years, the inclusion criteria required at least three months of treatment with a total dose of up to $100 \mathrm{~g}$ of $\mathrm{CPZ}$ equivalent per day - even though we have seen that an increase above $500 \mathrm{mg}$ produces no better results.

A second point is the specificity of the drugs themselves. We have classified the neuroleptics by taking into account their side effects; thus, autonomic side effects related to sedative compounds, and extrapyramidal side effects to some "polyvalent" or disinhibitory compounds. This conflicts with the concept of CPZequivalent. In a study of "severe" tardive dyskinesia, Gardos and his colleagues (1984) showed that more than $50 \%$ of cases had been caused by only two compounds: fluoperazine and thiothixene, which in our experience are considered to be particularly prone to induce parkinsonism.

To try to clarify the problem of the nature of the drugs, Deniker and Poirier employed a data bank of psychotropic drugs to propose an automatic classifica-

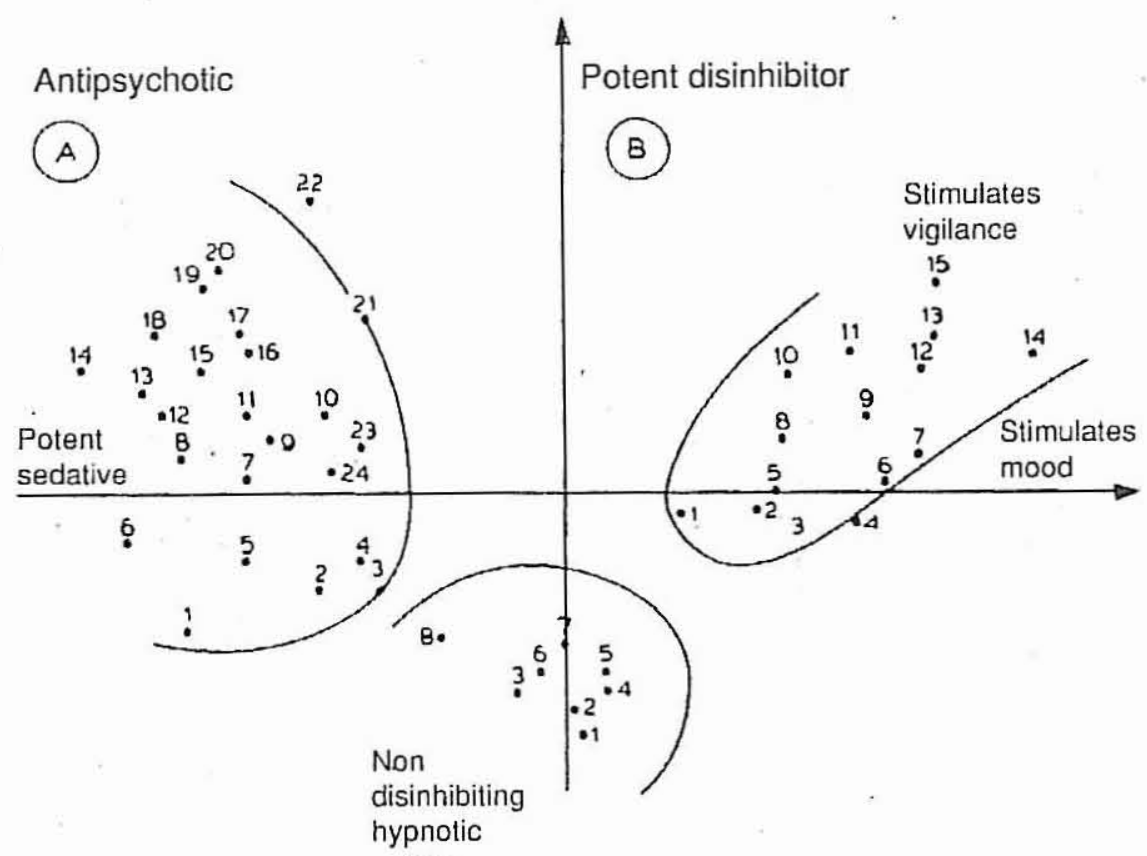

(c)

Fig. 4. An "automatic" classification of psychotropic drugs. Group A (antipsychotic): 1, reserpine; 2, cyamemazine; 3,levomepromazine; 4, chlorpromazine; 5, sultopride; 6, droperidol; 7,benperidol; 8, haloperidol; 9, trifluoperazine; 10, prochlorperazine; 11, perphenazine; 12, fluphenazine; 13, clothiapine; 14, fluanison; 15, clozapine; 16, oxaflumazine; 17,thioproperazine; 18, thiothixene; 19, trifluperidol;20, pipothiazine; 21, flupentixol; 22, penfluridol; 23, pimozide; 24, propericiazine. Group B (mood and vigilance stimulants): 1 , amitriptyline; 2 , trimeprimine; 3 , nortriptyline; 4 , maprotiline; 5 , imipramine; 6 , propizepine; 7 , isocarboxide; 8 , iproniazide; 9 , viloxazine; 10 , prolintane; 11 , pyrovaleron; 12,deanol; 13, fipexide ;14, cyprolidol; 15, d-amphetamine. Group C (sedative hypnotics): 1, mecloqualon; 2, vinbarbital; 3, phenobarbital; 4 , tofisopam; 5 , methaqualon; 6 , nitrazepam; 7 , diazepam; 8 , vinylbital. 
tion using statistical computerized methods. Multifactorial analysis of correspondences allowed us to make a definite separation of the cloud of neuroleptics from other psychotropic drugs. It is possible to distinguish two poles: below, the sedative compounds; and, above, the disinhibitors (Fig. 4) - as in the clinical, empirical classification shown in Fig.3.

Since at least 1983, it has been possible to explain the different properties of the neuroleptics by their different actions on the $D_{1}$ and $D_{2}$ postsynaptic dopaminergic receptors. Gardos had remarked that thioridazine seemed to have a "protecting" effect against severe tardive dyskinesia. Friedhof and his colleagues described an animal model of chronic tardive dyskinesia which showed that the best antagonist to the abnormal movements was sulpiride which acted on the $\mathrm{D}_{2}$ receptors. Unfortunately, this benzamide is not available on the American market, owing to its endocrine side effects such as amenorrhoea and galactorrhoea (the latter also affecting males). These endocrinological effects thus occur with a drug that is disinhibitory and a low inducer of extrapyramidal symptoms.

A final factor possibly implicated in the development of tardive dyskinesia concerns individual predisposition: mental retardation, organic damage, the elderly, etc. It is known, for example, that tardive dyskinesia can appear in aged women independently of chemotherapy.

\section{Neuroleptics and schizophrenia}

The antipsychotic actions of the neuroleptics were once considered as "anti-schizophrenic". After the discovery of the anti-dopaminergic action of these drugs, the hypothesis of a "hyper-dopaminergic" aetiology was proposed for this group of psychoses.

However, clinicians are well aware that chemotherapy is not an aetiological treatment for the psychoses since different forms can be identified and we prefer to speak of schizophrenias in the plural.

Initially, the neuroleptics are especially active against "productive" symptomatology such as excitation, hallucinations, and delusions. The early compounds are almost ineffective against the mood deficiency, social withdrawal and apathetic syndrome. With the introduction of drugs possessing disinhibitory properties, gradually some improvement was obtained in this regard. Yet the dopaminergic theory of schizophrenia retains little credibility for psychiatrists. However, Crow has proposed a double hypothesis: distinguishing between the "productive" and "defective" symptomatology of the clinical forms of psychosis, he correlates them with possible hyper- or hypo-dopaminergic dysregulation. This has still to be confirmed.

Paradoxically, these researches seem to have overlooked the early anti-manic specific activity included in the general definition of neuroleptics proposed in 1957. When the antidepressants were discovered, it appeared that they had the opposite effects to neuroleptics on mood regulation. Neuroleptics are effective against mania and, rarely, can induce some degree of depression (for instance, with reserpine). In contrast, antidepressants resolve depressive states and sometimes can produce euphoric reversion of mood. They have anti-reserpine activities, that is to say an anti-neuroleptic action.

Today, mood regulation is well known as a function involved not only in manic-depressive disorders but also in different kinds of psychoses such as dysthymic ones. This approach can be used sometimes to treat the hypothymic or athymic forms which are the most resistant of the schizophrenias.

\section{Requirements for the future}

In the case of mania, modern psychiatry cannot accept that excited patients make trouble in our quiet wards. So, it is important to have very rapidly acting tranquillizers - acting in an hour, like droperidol, but droperidol has hypotensive side effects and, in acute cases, the injection has to be repeated every 2 or 3 hours. Thus we need a fast neuroleptic with a prolonged duration of action.

In the chronic psychoses, the more or less "resistant" patients are of two types. The first have irreducible hallucinations or delusions, sometimes persisting unobtrusively. Neuroleptics have differing activities against hallucinations: the specific agents are the butyrophenones (especially haloperidol, triperidol and haloanisone), against flupenthixol and loxapine. Thus, there still remains a need in this special indication.

The second type of resistant psychoses are those in which there is a deficit of mood or social retardation. In many chronic schizophrenias both are present: persistent residual hallucinatory activity and loss of motivation and energy. Various means are available for combating this defective syndrome, for instance the use of more disinhibitory neuroleptics; the addition of antidepressants to the neuroleptic; or the use of compounds such as carpipramine, in which the molecule has a tricyclic nucleus and butyrophenone-like structure. From both the pharmacological and the clinical points of view, this is neither a regular neuroleptic nor an antidepressant, even if it is an effective stimulant drug, especially in the chronic residual schizophrenias. But within this chemical group are other compounds (such as clocapramine, the chlorinate compound) that are true regular neuroleptics.

Among the dibenzo-oxazepines, amoxapine has both antidepressant and neuroleptic properties. Even if it is given only for depression, extrapyramidal side effects may sometimes appear.

In summary, we have a present need of faster and longer acting effective tranquillizers, more effective anti-hallucinatory drugs and, above all, of disinhibiting antipsychotics. This latter type of drug (for example, piperazinic phenothiazines or butyrophenones) fre- 
quently induces extrapyramidal symptoms and can be expected to produce tardive dyskinesia if, the patient is not closely watched.

\section{Some new compounds}

Risperidone, a derivative of ritanserine, has the ability to block not only dopamine receptors, but also the serotonin receptor $5 \mathrm{HT}_{2}$. It is a completely new antipsychotic and unforeseen clinical applications may be expected.

Compounds blocking primarily the $\mathrm{D}_{2}$ receptors can be useful antipsychotics with a minimum of exIrapyramidal adverse elfects. Among the benzamides, amisulpricle has promising early antipsychotic actions and may have strong anti-hallucinatory properties.

Another benzamide, remoxipride, is also a $\mathrm{D}_{2}$ blocker with clear-cut antipsychotic action in the schizophrenias.

\section{Conclusion}

After 37 years, the neuroleptic story continues with new developments still to be examined. The face of madness
The neuroleptics: a historical survey<smiles>C1=C[C@H]2CC[C@H]12</smiles>

has been completely changed; not only by means of psychopharmacology but also by the development of psychotherapy, sociotherapy and the rehabilitation of the patients in the community. But this metamorphosis of the insane or lunatic into an ordinary patient has erased the clinically spectacular symptomatology of the past and we now have to keep our eyes and ears attuned if we are to observe the typical cases of psychopathology.

The modern lists of criteria and the different types of 'rating scales are necessary for communication between specialized centres and with other disciplines, yet we must not forget that we are psychiatrists attentive to human behaviour and relations, and not just accountants of quantitative methodology.

\section{References}

1. AYDFJ, BLACKWELL B. Discoveries in biological psychiatry. 1970. Lippincott: 253.

2. DENIKER P. From chlorpromazine to tardive dyskinesias. Psychiatr. J. Univ. Ottawa. 1989; 14: 253-259.

3. PARNHAM MJ., BRAINVELS J. Discoveries in pharmacology. Vol.1: Psycho- and neuropharmacology. 1983. Elsevier: 507.

4. JESTE DV, WYATTRI. Changing Epidemiolgy of tardive dyskinesia: an overview. Am. J. Psych. 1981; 138: 297-309. 